\title{
Helen Pritchard
}

\section{THINKING WITH THE \\ ANIMAL-HACKER: \\ ARTICULATION IN \\ ECOLOGIES OF EARTH \\ OBSERVATION}

APRJA Volume 2, Issue 1, 2013

ISSN 2245-7755

CC license: 'Attribution-NonCommercial-ShareAlike'. 
What happens if Nature is neither lacking nor primordial, but rather a plentitude of possibilities a cacophony of conversation? Indeed what if it is that same force field of articulation, reinvention and frission that we are used to calling - culture? (Vicki Kirby)

The 'eyes' made available in modern technological sciences shatter any idea of passive vision; these prosthetic devices show us that all eyes, including our own organic ones, are active perceptual systems (Donna Haraway).

\section{Prologue}

In the depths of the Cumbria hills a dairy cow changes its route to stare deep into the camera lens of the 'Environmental Virtual Observatory' (EVO) (www.evo-uk.org). Downstream at 15 minute intervals organic matter is pushed through turbidity probes, sometimes causing the computation to glitch and upload its own movement into a data storage warehouse. In this muddy, messy situation of the EVO there is something lurking, something which might be described as the 'Animal-Hacker' the non-human animal, an entity that exploits the computational ecology, reconfigures it in an act of what Donna Haraway would describe as "worlding" (92).

\section{The EVO}

The EVO is one of a number international projects that have emerged from the convergence of cloud computing, big data, remote sensing technologies, large scale government funding initiatives, the rising temperature of the earth and the co-evolving vision of a computational universe (Hayles 3 ). In networked observatory projects such as the EVO distributed sensors monitor and upload 'non-human' environmental processes and store them in the 'cloud'. The assumption is that we can use the 'gathered' data from earth processes (both live and archived) to analyze, predict, act and prevent 'changes' in the biophysical world. (Nold 3)

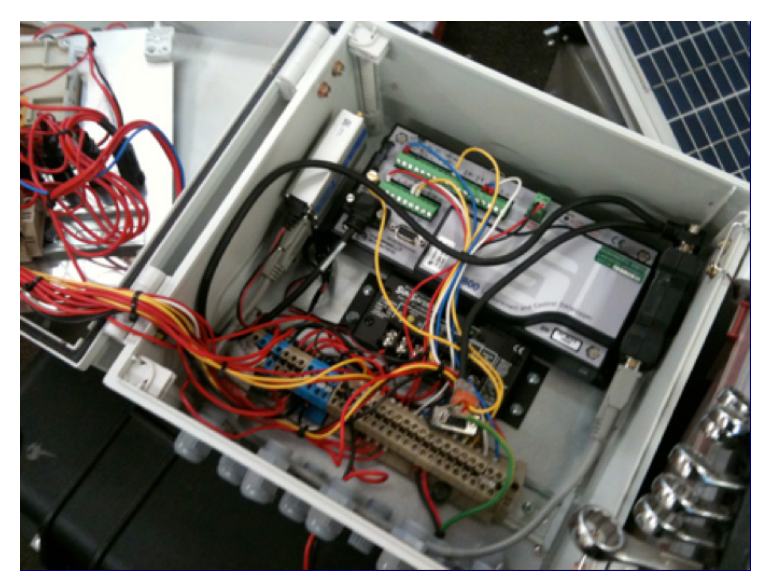

Image 1: Assembling sensing equipment in the Eden Dtc office (photo: Helen Pritchard)

This paper arises from embedded artsbased research in the EVO in order to grapple with the practices, sites and processes of Earth Observation. It emerged through a process of 'hanging out' (Pfaelzer 3)-talking, sharing resources, looking through microscopes, reading policy documents, lingering in cow sheds, lurking on social media sites, handling equipment, sharing long car journeys and downloading data sets.

Embedded arts-based research provides the method to inquire, interrogate and hold "apparatuses and the processes by which they are produced" (Barad 202). It shares many similarities with participant observation in feminist technoscience studies (Barad 202). It makes space for the researcher to 'do' with the instruments of interdisciplinary practice, rather that just observe them "to hold them to one's lips", to experience them through sensation, "like the 
smell of machinery grease, noxious chemicals, and other organic and inorganic matter" (ibid).

Through my initial inquiries I have experienced a mix of soggy samples, chemicals, mud, soldering irons, I have searched, rambled through lines of code in order to find a 'mistake', I have asked sunlight to write itself. I have held out my hand to the dripping nostrils of dairy cow and hung out during coding sessions to 'hold' incommensurable datasets, matter and ideas.

\section{New Materialist Approach}

In this short paper I attempt to build a framework on which to grapple with the entities of the EVO. I explore the provocation that nature writes itself into the folds of computation and collective becoming, drawing on New Materialist work of Haraway, Barad and Kirby. The New Materialist approach to practices of 'earth observation' provides a theoretical framework for witnessing and exploring human and non-human practices, interactions, knowledges and affects. In the context of research on computing ecologies it provides a way to grapple with "the material artifacts and natural stuff that populate our [computational] environments as well as on socioeconomic structures that produce and reproduce the conditions of our everyday lives" (Coole 1). The methodologies of New Materialism evoke the more-than-human agencies, knowledges and politics that circulate through inquiry of material realities. They make space to consider the non human writer, the 'Animal Hackers'.

By examining the matter and discourse of 'Earth Observations', that are enabled by network technologies, cloud computing and sensors, such as networks of remote sensors, pulsating live data sets, mud covered cameras, networked animals and computational imaginaries, I aim to foreground nonhuman forces in these assemblages. The 'non-human' forces I am referring to include nonhuman animals, plants, watercourses, earth energies as well as hardware and software .

There has been a surge in applying 'New Materialist' thinking to technological assemblages that place emphasis on nonhuman forces. However, my concerns are specifically in recognition of the drive towards planetary-scale computation and the wider imaginary of nature-objects in the 'internet of things'. My aim is to make apparent the relative invisibility of non-human forces/writers in these assemblages and to work towards developing a set of practices as a manifesto for 'More-than-human' collective computing.

\section{The EVO}

The EVO is a proof of concept project and exemplar of the drive towards what has been described as 'planetary scale computation' (Bratton DG.P) and 'computational planetary skins' (Stepney 3). As Bratton explains the practice of planetary scale computing is both the distribution of large amounts of data across "far-flung data centres" together with "the layering of software and hardware across a multitude of scales from "cloud computing to addressable nano bots". In the EVO the planetary-scale computational vision is that layers of hardware and software are spread across multiple sites and entities. Large data sets are stored in data centres and automatically moved and replicated through computation without human intervention. These layers form what Donna Haraway might describe as profound reconfigurations of bodies and processes; as both human and non-human bodies are entangled in computational 
practices. In the EVO Dairy Cows staring down remote cameras or peaks in river flow bring us "face-to face [through the network] with significant others" (93). If we consider these events not as measuring or writing the other, but instead as co-writing with articulate non-humans, "Textual Adventures"(Kirby 76) then the question arises of how we might think with and from non human animalwriters in order to "speculate, imagine, feel, build something better"(Haraway 92). In this paper I tentatively introduce the figure of the 'Animal-Hacker' to consider the articulation of nonhuman entities in these computational ecologies.

\section{Non-Human Coders}

The 'Animal-Hacker' is a proposition to consider within the context of 'environmental observation' Haraway's call to "open up the question of non humanism" (92). To breakdown existing abstractions of nature and computation in order that "richer, more responsive inventions, speculation and proposing - worlding- can go on" (93). Franco "Bifo" Beradi writes in the foreword to Geoff Cox's book 'Speaking Code' that "the effects of code are not [as might be assumed] deterministic, as far as code is the product of code writing, and code writing is affected by social, political, cultural, and emotional processes" $(x)$. Therefore code writings in the EVO can perhaps be considered in Haraway's terms as "relational knots" the "coproduction" of knowledge"and a "becoming with" that occurs as two "things" (animal and code) have an encounter. In this proposition 'Hacking' code, might be considered as new "worldings" or as Beradi describes "lines of escape". ( $\mathrm{x}$ )

The familiar co-constituted animals that appear in computational ecologies such as the EVO are productive agents who contribute vital affordances of one kind or another. As Kirby would describe they are articulate and write themselves through a variety of instruments, translations and representations (81.) However, these articulations, these interferences with computation, are not always compliant. In this muddy, messy situation of the EVO I have experienced something which might be described as the 'Animal-Hacker' the non-human animal, an entity that exploits the computational ecology, reconfigures it in an act of worlding. In my tentative observations, entangled entities, such as cows, diatoms, owls and plants articulate themselves both through compliance with, and disruption of, the computational architecture that has been laid down 'for' them.

If we address the 'Animal-Hacker', not as a passive object of observation, but as co-creating computational environments, how might we consider the non human animal? If we are serious about forms of engagement with non humans, can we engage with the 'Animal-Hacker' as a possible invitation to reconsider a possible introduction to other-worlding?

\section{Environmental Observation}

To date, the majority of research in 'Environmental or Earth Observation' has focused on the deployment of the technologies (as observed in Gabrys ) both through large-scale government initiatives (Teillet et al) and via localized, citizen sensing and so-called DIY projects (Cuff 3). Concurrent research in the areas of histories of earth observation (Dourish), media archaeologies of ubiquitous computing (ubicomp), weather systems, earth observation and sensor technologies has broadened the context for this research project. Recent research 
has considered how ubicomp might inform parallel sensing practices through distributed sensation and experience (Hansen, Gabrys) and interspecies sense-making (Mancini, et al) the 'Animal-Hacker' project engages with these bodies of work to consider articulation distributed across agents and entities. There is also a rapidly increasing amount of work on coupling computational earth observation systems with cloud-based services, described. These cloud-based services will be used for remote trading, with trades based on computational measurements of the non human 'living'. One example is the implementation of water trading in which fresh clean water and river flows will be measured through remote sensing, calculated and sold to both individuals and corporations within the cloud infrastructures (Marshall ).

Existing research on environmental observation emerges both as inter-disciplinary and/or located in a number of areas of research for example Computing, specifically, Human-Computer-Interaction (Bell \& Dourish, Stepney) Design \& Geopolitics (Bratton) Software studies and Critical Engineering (Gabrys, Fuller, Chun \& Hui, Nold), Media Theory and Design. My work does not intend to polarize the different approaches of science and humanities to environmental observation, but rather, by drawing on the work of feminist technoscience (Haraway, Suchman) and ecological methodologies, attempts interdisciplinary arrangements.

\section{Planetary Skins}

As a proof of concept project and prototype the EVO is part of and produces the the vision for the 'internet of things', as part of the imaginary of ubicomp (Weiser). Outlined in 1999 the vision for ubicomp or calm computing was a world of serenity in which technology was to keep us "perpetually informed of what is happening around us, what is going to happen and what has just happened". The EVO is a project informed by Weiser's vision, with the aim to use ubicomp and remote sensing to inform us of earth processes and non human activity in order to create warning systems and comfort.

As a 'proof of concept' project the EVO both enacts the processes of environmental observation and imagines its future practice. The EVO is also knotted with a deeper history, the desire to expand human sensory capacities (Hansen 2). This imagination revolves around making the 'whole' of human and non-human environments legible for computer systems (Nold).

It enacts both the means and the metaphor of what Katherine Hayles describes as the 'Computational Universe' (3). For Hayles this universe is one in which we make and imagine the universe through the lens of our own computational age.

The computation of non-human environments in computer science or 'natural computation' is an ambiguous term. It refers to the space at the intersection of 'nature' and computation. Wikipedia defines natural computation as a terminology that was introduced to encompass three classes of methods (wikipedia.org). Those that take inspiration from nature for the development of novel problem-solving techniques, such as bio-inspired software or evolutionary algorithms. Secondly, methods that use computers to 'synthesize natural phenomena' and, thirdly, those methods that employ natural materials (eg. Molecules) to compute.

However, as in Hayles' 'Computational Universe' there is also a dual co-evolving aspect to 'natural computation', namely, the drive towards "understanding nature as information processing". This understanding draws on the work of physicists such as Stephan Wolfram who claim that the 
universe is generated through computational processes running on a vast computational mechanism underlying all of physical reality (15). Bratton explores the notion of the world as made up of discrete units, referencing thinkers such as Wolfram. He notes that these diverse and complex theories trickle down to simplified and widespread sensibility that "the world is a computer and the best way to listen to that computer is with other computers" (15). The layer of computational technologies smeared over the planet is just a way to get closer to a primordial digital unfolding of all things (ibid). For others, such as Alex S. Taylor at Microsoft Research, what is crucial is that, as work develops at the intersection of the biological, geological and the computational, inhuman nature that fails to 'register' within the computational regime, will become excluded from systems of recognition. His concern is that nature that cannot be computerized will no longer be recognized as nature.

\section{Close up Mess}

'Environmental Observation' through remote sensing, big data and cloud computing is the coming together of a number of computational systems which are part of the vision of ubicomp. Alongside the myth of ubicomp is the practical reality of working with these technologies day to day. Bell and Dourish use mess to suggest that practices of technology are never quite as simple, straightforward or idealized as we might imagine them to be. For any infrastructures, the mess, Bell and Dourish argue, is never very far away. Mess is both the matter of these technologies the "mazes of cables, the connectors, clips, clamps and duct tape" and the productive discourses "the regulatory authorities who authorise intervention, governments that set policy, bureaucrats" (Bell \& Dourish 1). In contrast to the vision of ubicomp as a slick system it "looks" very different (Bell \& Dourish in Anderson \& Pold 2). As Anderson \& Pold observe "Ubicomp has developed as a messy cultural interface rather than a seamless tool for work". (2)

The messy practices of sensing technologies are also explored in the work of Antti Oulasvirta in when "Users do the Ubicomp". Oulasvirta argues that ubicomp can be viewed from two distinct perspectives, on one hand there is the avant garde of ubicomp that gets presented at conferences, a conceptualization that draws on visions from Mark Weiser and others. On the other hand is what she describes as "the real ubicomp" a massive non-centralized agglomeration of devices, connectivity, electricity means, applications, services and interfaces, as well as material objects such as cables, meeting rooms and support surfaces that have emerged anarchically. In the EVO this also includes other agents such as nonhuman animals, plants, forests, rivers, fences, muddy puddles and cow feaces.

These infrastructures are fragmented, and across practices, technologies are lashed together (Dourish and Bell 1). Oulasvirta argues that this often means that technologies are affected by seemingly remote factors. This is apparent in the EVO where everyday practices are affected by policy, funding, laws on data storage, fragmented data sets, theft of equipment and the energy of non-human forces, such as sediment contaminating readings or an owl's wing blocking the webcam. In some ways the ecologies of the EVO are as complex, as the ecologies it seeks to 'observe'. 


\section{From 'Sensemaking' to Sensation}

One of the key themes in ubicomp and earth observation is that of "Sensemaking" through computation. In the case of earth observation the term draws on the $\mathrm{HCl}$ definition (Russell, Stefik, Pirolli, \& Card) and is used to describe "making sense of the world using information technology". As described in a special issue for ACM "'Sensemaking' involves collecting, organizing and creating representations of complex information sets, all centered on the formation and support of mental models involved in understanding a problem that needs to be solved" (Pirolli et al, 1). The ACM journal cites examples of such problems including "understanding a health problem to make a medical decision, understanding the weather to make a forecast and intelligence analysis to identify strategic threats" in the case of the EVO, the problem to be understood is that of water pollution and climate change "the EVO faces the challenge of finding and making sense of environmental data" (evo-uk.org). In the positivist paradigm, 'Sensemaking' is premised on understanding through discovery. The positivist understanding of "Sensemaking" in much computing literature leads to a focus on the algorithms and technologies of sensing. The notions of "collection" and "organization" can be recognized as prevalent in the literature and in the technologies developed for earth observation.

The concept of 'Sensemaking' is deeply embedded within the regime of the computational universe as the engineering of remote sensor networks and the positivist conceptualization of 'Sensemaking' continuously inform each other in a series of feedback loops. As Kathryn Yusoff explains an 'understanding of how sense is enrolled into our habits of thought and theories of materialities is crucial if we are to create new practices of sensations and new sensibilities" (2) In the positivist framework the desire to makediscover the world positions the 'thing' being sensed in this case as an object rather than a process. It perpetuates a human-centered understanding of the 'environment' which is recognized within these schemes as passive, discoverable and accessible. Environmental sensing in this paradigm assumes that 'nature' exists in discrete units, units which can be measured, organized and made sense of. 'Sensemaking' becomes an important part of the computational regime, and in itself a way to articulate other activities. So much so that even citizen scientists become rebranded as "Sensemakers" human bodies extending the sensor network, such as in the website $\mathrm{http}: / /$ sensemake.rs for their "air quality egg project".

This particular 'Sensemaking' approach to remote sensing obscures what is revolutionary about the complex dynamic of environmental sensing and big data. Which is not simply a development of remote sensing and data crunching, but is as research from Post Humanities suggests (notably Chun, Gabrys, Hayles and Hansen) the overlapping or imbrication of technics and sensation. This overlapping can be understood as Gabrys describes as significantly extending the distribution of sensation (5) and collective becoming.

\section{Collective Life}

In the particular case of the EVO, computation is part of the reconfiguration of entities, the formation of space for collective life and the organization of communities. A common theme in post-humanities work on Earth Observation is the conceptualization of 


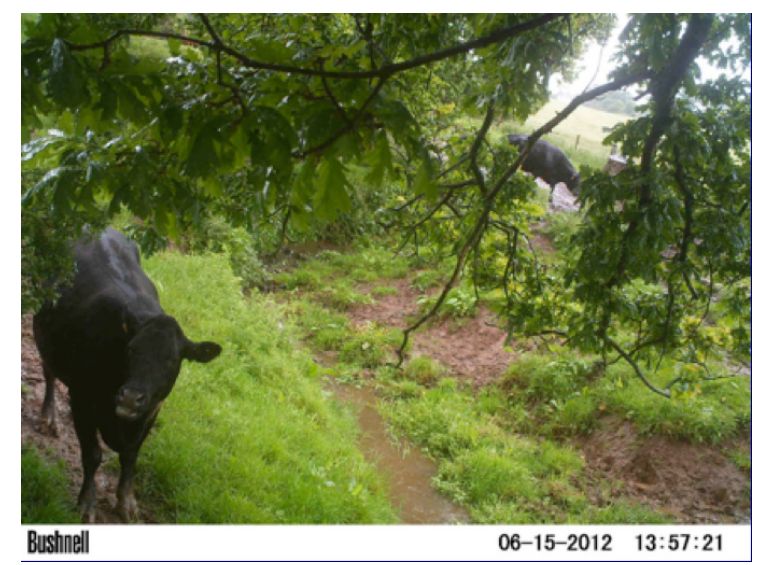

Image 2 : Cow address 1: Automated image from a Bushnell motion detector camera, (2012)

sensing as a form of questioning, a codely call to an active agent rather than the observation of a passive subject (Bratton \& Jeremijenko 21 , Bassett in Jones 200). This might also be described in terms of computation as questions thrown across a space in the form of energy and a response bounced back (200). In Basset's work remote sensing is approached through vision and touch through the network, remote sensors are explored as an assemblage that make it possible to touch a surface to interrogate it from a distance, without being in direct contact with it. However, Bassett argues that this touch is asymmetrical and without haptic clues. For Basset the significance of remote sensing is not in terms of the information, the messages that the remote sensing interactions carry, but instead the 'affect' they have. It is the not the message (meaning) but the energy (affect) that is sent and received that provokes a response. In this paradigm sensing is a process of affect.

In 'Sensing an Experimental Forest' Gabrys (2) invokes the theoretical perspective of Isabelle Stengers on Alfred North Whitehead to discuss the composition of sensing as a merging of experiment and experience. Gabrys considers sensors not as "Sensemaking' tool" "sensing something out-there" but instead as devices that make present and interpretable ecological processes. (2). Gabrys describes computational process as drawing together "experiencing entities" (2) that inform new arrangements of environmental sensing. These new arrangements are the new kind of science of big data, one which is fragmented, contingent, distributed "new worldings". Gabrys reconceptualises the biophysical world as an active entity which becomes present through technological arrangements, rather than a passive object awaiting measurement.

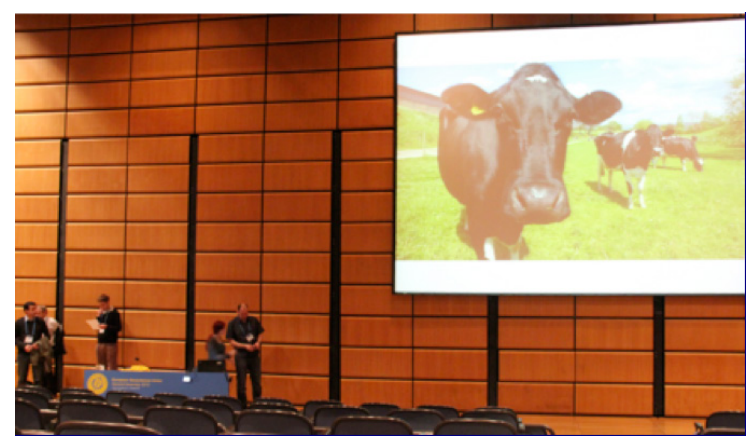

Image 3 : Cow address 2 : Catchment science, management and stakeholder participation

\section{Worldly Configurations}

In the EVO the computation of 'nature' provides an intimate, pervasive and profound reconfiguring of bodies and processes (both human and non-human). Computation is an entanglement of apparatus and entities. Understood through Barad's agential realism, computation does not allow us to observe the earth neutrally, nor does it only constrain what we see, rather it "helps produce and is part of" the earth-body it images ("Meeting the Universe"101). The thoroughly distributed, networked and embedded multitudes of computational entities from which Earth observation emerges give the world a specific material form, creating 'specific worldly configurations which in turn make 
knowledge' ("Posthumanist Performativity" 393). The processes effect "what's real and what's possible, as some things come to matter and others are excluded, as possibilities are opened up and others are foreclosed" (ibid) .

Who, and what, participates in the computational worldings of earth observation is a question of ethical, political and ecological urgency. It concerns Haraway describes as "who and what are to be forged" ("staying with the trouble"). For Haraway the forging "is the biological cosmopolitical practice of articulating bodies to other bodies" (ibid) the importance is that these practices are done with care so that significant others might flourish".

\section{Exploits and Hacks}

The promise of ubiquitous computing, remote sensing, environmental observation has been to make the 'invisible visible' (Cuff \& Hansen 2). The whole purpose of these systems is to script some sense of order into the world (Bratton "Post Oil World" 8). The layering of measurement, observation, listening, speaking, of big data and cloud computing, creates a possibility space of information on how wordly systems perform and relate (8). To monitor the earth through remote sensing has an impact on culture that is similar in scale and complexity as the invention of the microscope (Hansen 2). To model (through the use of distributed computing power) the interrelationships of complex ecologies, is to "open up the complexity and agency of worlds we could not imagine" (ibid). Earth Observations claims to expand vision-making and, as a result, make new domains of sensation accessible to human experience. The promise of making the invisible visible positions the 'thing' being sensed/ made visible as an object. It perpetuates a human-centered understanding of the 'environment' which evokes schemes as passive, discoverable and accessible.

Haraway describes this as a political practice. In the emerging knowledge systems of contemporary earth observation, there is a similar question of how we regard the material practice of computing and the way we labor on, exploit and interact with nature. What is at stake is participation within nature and the collective productions of meaning and affect. As Barad would say what is in question is the nature of 'nature' (67). How we are 'done, undone or redone' ("staying with the trouble") through our collective becomings of worlding in the "conjoined flesh of multispecies tangles". (ibid)

A material account of sensing technologies highlights a particular tension with the promise of making visible the invisible. As Chun explains, the idea that information [computation] makes the invisible visible is in conflict with the actual operations of computation. As, for "computers to be a machine that makes things 'transparent', the fact that they 'compute', that they generate images, models and texts, rather than merely represent or reproduce what exists elsewhere must be forgotten" (1).

However, this does not mean that we must assume that the 'computation of nature' is an interpretation, is an illusion of a world that cannot be accessed. Because as Kirby suggests we are well-aware that data is indicative, that it "throws up nodes of reference that effectively correspond" (Latour 24 cited in Kirby 81). The process of articulation is very different to the act of making the 'invisible visible'. The articulation of experiencing entities in computational systems emerges through intra-actions, entanglement between component parts entities, between the 'measured object' and the 'measuring device"' (Barad 337). If we reconsider the participation of 
living matter in computational ecologies as not just something which is 'sensed', 'measured' 'written', or even 'written with' but rather as itself, simply writing, then how might we enact worldings that care for, learn with and from the 'Animal-Hacker'? How might we think with the 'Animal-Hacker' to rethink the roles of non-human participation in practices of earth observation, computation and collective becoming?

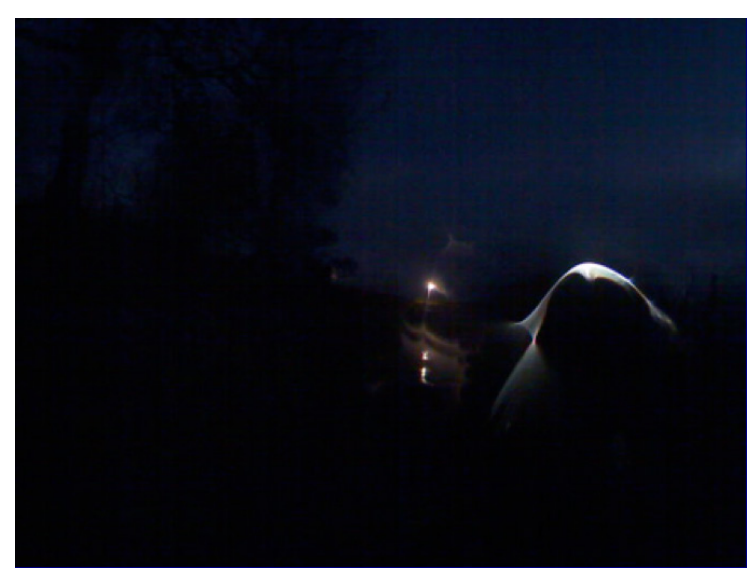

Image 4 :Animal Hacker: Owl intervention:

Automated image from timelapse webcam, Eden DTC (photo courtesy of Eden dtc)

\section{Acknowledgments}

Thank you to the EVO project. Also thanks are due to my supervisors Gordon Blair, Emile Devereaux and Kathryn Yusoff. Also I am grateful to Jane Prophet for reading an earlier draft and to Geoff Cox, Yara Guasque, Tatiana Bazzichelli and Søren Pold for their feedback at researching BWPWAP. Also thank you to the other participants of researching BWPWAP for their feedback during the workshop and to Winnie Soon. This paper was developed as part of postgraduate studies at HighWire, Lancaster University. RCUK Grant EP/G037582/1 and during visiting research at the School of Creative Media , City University, HK. 


\section{Works cited}

Albaladejo, Cristina et al.. Wireless Sensor Networks for oceanographic monitoring: a systematic review. Sensors, Basel, Switzerland, 2010 10(7), pp.6948-68

Andersen, Christian and Søren Pold. The Scripted Spaces of Urban Ubiquitous Computing: The experience, poetics, and politics of public scripted space, Fibreculture Journal, 2011(19), 110-125.

Barad. Karen. Posthumanist Performativity: Toward an Understanding of HowMatter Comes to Matter. Signs: Journal of Women in Culture and Society 2003 28(3)

Barad, Karen. Meeting the Universe Halfway: Quantum Physics and the Entanglement of Matter and Meaning. Durham: Duke UP, 2007

Bell, Genevieve \& Paul Dourish. Yesterday's tomorrows: notes on ubiquitous computing's dominant vision. Personal and Ubiquitous Computing, 2006 11(2), pp.133-143.

Bratton, Benjamin \& Natalie Jeremijenko. Situated Advocacy, Situated Technologies Pamphlet Series, IDC, 2008

Bratton, Benjamin. Talk: Sea and the Cloud, available at http://vimeo.com/41825055 2011

Chun, Wendy Hui. On Software, or the Persistence of Visual Knowlegde. Grey Room, Inc., 2012 18(18), pp.26-51.

Cox, Geoff. Speaking Code: Coding as Aesthetic and Political Expression MIT Press 2013
Cuff, Dana \& Mark Hansen. 'Urban Sensing: Out of the Woods,' Communications of the Association for Computing Machinery 51, no. 32008

Dourish, Paul et al.. Ubicomp's colonial impulse. Proceedings of the 2012 ACM Conference on Ubiquitous Computing UbiComp 2012 12, p.133.

Jones, Caroline. Sensorium: Embodied Experience, Technology, and Contemporary Art. MIT Press. 2006

Gabrys, Jennifer. Sensing an Experimental Forest, Computational Culture Vol 2. 2012

Hansen, Mark. Ubiquitous Sensation or the Autonomy of the Peripheral: Towards an Atmospheric, Impersonal and Microtemporal Media, in Throughout: Art and Culture Emerging With Ubiquitous Computing, edited by U. Ekman 2012

Haraway, Donna. Modest Witness@ Second Milennium. FemaleMan Meets OncoMouse. New York: Routledge. 1997

Haraway, Donna. When Species Meet University of Minnesota Press 2007

Haraway, Donna. Staying with the Trouble, Xenoecologies of Home for Companions in the Contested Zones, http://www.culanth. org/?q=node/353 2010

Jahn, Marisa. Byproduct: On the Excess of Embedded Art Practices, Yyz Books, 2010

Kirby, Vicki. Quantum anthropologies: Life at large. Durham [N.C.]: Duke University Press.2011

Kirby, Vicki. Telling Flesh: The Substance of the Corporeal. New York \& London: Routledge, 1997 
Mancini, Clara. Exploring interspecies sensemaking: dog tracking semiotics and multispecies ethnography Conference Item Exploring Interspecies Sensemaking : Dog Tracking Semiotics and Multispecies Ethnography.(2012)

Marshall, Christa. Will Water Become the Chief Commodity of the 21st Century?, Climate Wire, (http://www.scientificamerican. com/article.cfm?id=will-water-become-thec...) 2012

Nold, Christian \& Rob van Kranenburg. INTERNET OF PEOPLE FOR A POSTOIL WORLD, The Situated Technologies Pamphlet Series, IDC 2011

Oulasvirta, Antti. "When Users Do" the Ubicomp, 2007 pp.6-9.

Pfaelzer, Jean. Hanging Out: A Research Methodology. Legacy: A Journal of American Women Writers, 27(1), 2010 pp.140-159.

Stepney, Susan \& John Clark, J, Harnessing the Planetary Computational Skin. 2006

Suchman, Lucy. Feminist STS and the Sciences of the Artificial. In: New Handbook of Science and Technology Studies. MIT Press. 2007

Summerhayes, Caroline. EMBODIED S PACE IN G OOGLE E ARTH, III(1), 2011 pp.113-134.

Weiser, Mark. Pervasive Computing. 2003 pp.43-49.

Weiser, Mark, Rich Gold and John Seely Brown (1999). "The origins of ubiquitous computing research at PARC in the late 1980s". IBM systems journal 38 (4): 1999 693
Yusoff, Kathryn. 'Insensible worlds: postrelational ethics, indeterminacy and the (k)nots of relating' Environment and Planning D: Society and Space. 2012 